\title{
Electricity-free amplification and visual detection of Cronobacter species in powdered infant formula
}

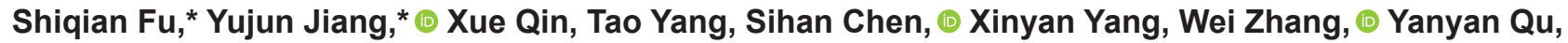 \\ and Chaoxin Man† (i) \\ Key Laboratory of Dairy Science, Ministry of Education, College of Food Science, Northeast Agricultural University, Harbin 150030, China
}

\section{ABSTRACT}

Due to the lack of electricity and thermostatic instruments in certain settings for convenient detection of Cronobacter species in powdered infant formula (PIF), a novel investigation was conducted to establish an electricity-free visual detection system for rapid detection of Cronobacter species in PIF. This system included a portable electricity-free heater that could use the exothermic reaction of calcium oxide and water and 3 kinds of phase change materials to supply 3 constant temperatures for immunomagnetic separation, DNA extraction, and loop-mediated isothermal amplification assay. Meanwhile, the amplified reaction combined with hydroxynaphthol blue could achieve rapid visual detection. Primers designed based on the 16S-23S ribosomal RNA internal transcribed spacer were used in loop-mediated isothermal amplification to specifically monitor Cronobacter species, and the detection limit can reach $4.2 \times 10^{2} \mathrm{cfu} / \mathrm{g}$ in PIF by an electricity-free heater in $2 \mathrm{~h} 30 \mathrm{~min}$. Moreover, $2 \mathrm{~h}$ of pre-enrichment was necessary when the level of the PIF samples with Cronobacter spp. was $10^{0} \mathrm{cfu} / \mathrm{g}$. The stability of the system was evaluated in ambient temperature at $4^{\circ} \mathrm{C}, 25^{\circ} \mathrm{C}$, and $37^{\circ} \mathrm{C}$. The results suggested that the electricity-free heater can maintain 3 constant temperatures to support different processes. Therefore, this amplification and visual system is applicable for use in many fields for rapid and specific detection of Cronobacter species in PIF.

Key words: electricity-free visual detection, immunomagnetic separation, loop-mediated isothermal amplification, powdered infant formula, Cronobacter species

Received September 26, 2019.

Accepted March 9, 2020.

*These authors contributed equally to this work.

$\dagger$ Corresponding author: mcxwh2006@qq.com

\section{INTRODUCTION}

Cronobacter spp., previously called Enterobacter sakazakii, mainly infect newborns and infants and can cause meningitis, necrotizing enterocolitis, and bacteremia with a fatality rate of about 40 to $80 \%$ (Healy et al., 2010; Yan and Fanning, 2015). Powdered infant formula (PIF) has been recognized as the main source of infection (Song et al., 2016), and the delayed detection and report of contaminated pathogens in PIF could cause outbreaks of foodborne disease (Norberg et al., 2012). Consequently, a simple and rapid detection method is extremely important in resource-limited settings. The immunochromatographic strip has been widely applied to detect pathogens because of its simplicity, rapidness, and no need for equipment (Huang et al., 2016; Luo et al., 2017), whereas it has some limitations in sensitivity and specificity. Nucleic acid detection as a rapid detection method is very efficient for detecting pathogens. However, most nucleic acid detecting techniques have been restricted to laboratories rather than the limited conditions where need is foremost (Pecchia and Lio, 2018).

Loop-mediated isothermal amplification (LAMP) is maintained at $65^{\circ} \mathrm{C}$ for $1 \mathrm{~h}$ and has high specificity and sensitivity without trained persons, costly equipment, or thermal cycles (Yan et al., 2014; Yin et al., 2016); the operation process is simple and much less sensitive to inhibitors than PCR assay (Zhao et al., 2017). Furthermore, LAMP products can be observed by direct turbidity, fluorescent dye, and immunochromatographic strips (Singleton et al., 2014; Wang et al., 2017).

The LAMP assay requires a heat source to complete amplified reaction. To eliminate the demand for electric instruments in limited conditions, some electricity-free heaters have been designed to maintain temperature of LAMP reaction in recent years (Labarre et al., 2010; Sema et al., 2015). These electricity-free heaters use the heat release of chemical reactions combined with phase change material (PCM) to regulate temperature (Labarre et al., 2011; Liao et al., 2016). For example, 
Curtis et al. (2012) designed a noninstrumented nucleic acid heater for point-of-care detection of HIV-1, and the LAMP assay for detection of $E$. coli has been conducted using a self-heating cartridge (Liu et al., 2011). Additionally, the analysis of on-site detection revealed that LAMP products integrating with these electricityfree devices could be simultaneously detected by immunochromatographic strip and fluorescent dyes to obtain visual detection results (Hatano et al., 2010; Singleton et al., 2014). However, all aforementioned devices lack the capabilities of bacteria pre-enrichment and DNA extraction, despite various DNA extraction methods that have been developed (Abdelhai et al., 2016). These methods are generally conducted in the laboratory; for example, centrifuge procedure for concentration of the bacteria requires electricity, and could not be completed in limited conditions. Magnetic nanobead-antibody complex (MNB-Ab) has been applied to perform immunomagnetic separation (IMS) in samples (Wang et al., 2011; Sun et al., 2015), which could selectively concentrate target bacteria at a constant temperature. Hence, IMS combined with LAMP assay is more suitable for electricity-free detection of pathogenic bacteria.

In our investigation, an electricity-free heater as reaction equipment that uses 3 kinds of PCM to regulate different constant temperatures was introduced for rapid detection of Cronobacter spp. in PIF. The process was divided into 3 steps including the IMS, DNA extraction, and LAMP-hydroxynaphthol blue (HNB) assay. The HNB was added into the reaction mixture in advance to avoid contamination and achieve visual detection. Thus, an electricity-free amplification and visual detection system was established for Cronobacter spp. in PIF in resource-limited settings.

\section{MATERIALS AND METHODS}

\section{Portable Electricity-Free Heater}

The portable electricity-free heater we designed obtained heat resource from the exothermic reaction of calcium oxide and water, and the schematic diagram is shown in Figure 1. Three kinds of PCM (Rubitherm, Berlin, Germany) with different heating capacity and melting temperature were used to maintain required temperature for the whole detection process, and the PCM maintained temperatures of $35^{\circ} \mathrm{C}, 65^{\circ} \mathrm{C}$ and $100^{\circ} \mathrm{C}$, respectively, which contributed to the steps of IMS, DNA extraction, and LAMP reaction. A vacuum cup was employed for thermal insulation. The heater consisted of 2 chambers, one containing the exothermic reaction and the other containing an aluminum box with PCM, which had a hole in the center. The size of hole was suitable for a $200-\mu \mathrm{L}$ PCR tube and $2-\mathrm{mL}$ epoxy epoxide tube, respectively, and the different steps in the same electricity-free heater were conducted in the process of detection by selecting the aluminum box with the desired size of hole and adding different weights of calcium oxide and water, combining with PCM for the required melting temperature. Then the tube was embedded in PCM through the hole. One type K (Fluke Process Instruments, Shanghai, China) thermocouple connected with a digital thermometer was inserted in PCM which can measure reaction temperature in real time.

\section{Preparation of MNB-Ab}

The mouse anti-Cronobacter spp. monoclonal antibody (Shanghai Huiyun, Shanghai, China) was conjugated with carboxyl magnetic nanobeads (MNB)
A

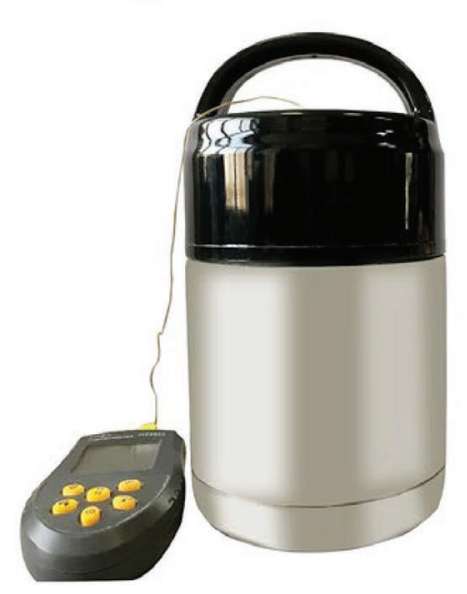

B

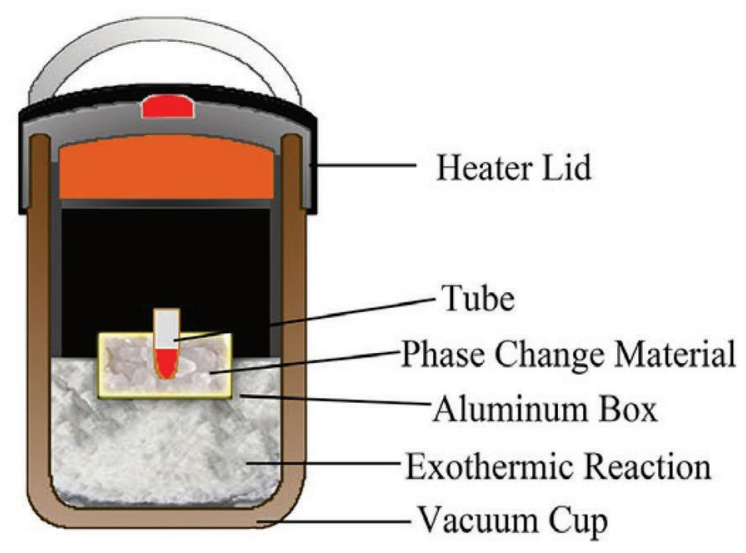

Figure 1. Schematic diagram of the portable electricity-free heater. (A) The appearance of the electricity-free heater. (B) The transection of the internal structure of the electricity-free heater. 
forming covalent conjugates via amide bonds using carbodiimide hydrochloride according to the manufacturer's instruction (Sigma, St. Louis, MO). The preparation procedures of MNB-Ab are described as follows: $50 \mu \mathrm{L}$ of carboxyl MNB $(10 \mathrm{mg} / \mathrm{mL})$ was washed with $560 \mu \mathrm{L}$ of $2-(N$-morpholino)ethanesulfonic acid (MES) solution ( $\mathrm{pH} 6.5,15 \mathrm{mM}$ ) and magnetic separation for $2 \mathrm{~min}$, then supernatant was removed. The process was repeated 3 times. Then, carboxyl MNB were suspended in $400 \mu \mathrm{L}$ of MES solution, and underwent ultrasonic treatment for $10 \mathrm{~min}$ at $200 \mathrm{~W}$, and magnetic separation for $2 \mathrm{~min}$. The supernatant was removed and the sediment was suspended again in $400 \mu \mathrm{L}$ of MES solution. Then $60 \mu \mathrm{L}$ of carbodiimide hydrochloride solution $(10 \mathrm{mg} / \mathrm{mL})$ was mixed and incubated at room temperature under shaking for $30 \mathrm{~min}$; next, the mixtures were washed 3 times using MES solution and MEST solution (15 mM MES, $0.02 \%$ Tween 20, pH 6.0 ), respectively, followed by magnetic separation and removing the supernatant. Subsequently, the carboxyl MNB were blended with $400 \mu \mathrm{L}$ of MEST solution and $80 \mu \mathrm{L}$ of antibody $(2.6 \mathrm{mg} / \mathrm{mL})$ to incubate for $12 \mathrm{~h}$ at room temperature under shaking so that the antibody could link with carboxyl MNB; then, the mixtures were washed 5 times using $400 \mu \mathrm{L}$ of PBST solution $(10 \mathrm{mM}$ PBS, $0.02 \%$ Tween 20, pH 6.0) and $400 \mu \mathrm{L}$ of PBS solution (10 $\mathrm{m} M, \mathrm{pH} 7.3)$, respectively, to wash away the antibody that did not conjugate with carboxyl MNB. After magnetic separation and elimination of supernatant, the sediment was finally resuspended in $500 \mu \mathrm{L}$ of PBS solution as MNB-Ab for IMS.

\section{Bacterial Strain Culturing and Sample Preparation}

A total of 44 bacterial strains including 19 Cronobacter strains and 25 non-Cronobacter strains are listed in Table 1. All Cronobacter strains were cultured in 20 $\mathrm{mL}$ of trypticase soy broth at $37^{\circ} \mathrm{C}$ for $14 \mathrm{~h}$. The other 25 bacterial strains were incubated in nutrient broth at $37^{\circ} \mathrm{C}$ under shaking (200 rpm) for $14 \mathrm{~h}$ to extract DNA. Cronobacter sakazakii ATCC 29544 was used as a sample to detect the sensitivity in PIF and the stability of the device. To determine the concentration of $C$. sakazakii, $100 \mu \mathrm{L}$ of serial 10-fold dilution of enriched C. sakazakii was added to trypticase soy agar plates and incubated at $37^{\circ} \mathrm{C}$ for $18 \mathrm{~h}$ before enumeration. The PIF samples purchased from supermarkets were tested according to the method of the US Food and Drug Administration to determine that the samples were not contaminated with Cronobacter spp. (Kim et al., 2016). Then $10 \mathrm{~g}$ of PIF was homogenized in $90 \mathrm{~mL}$ of sterile PBS solution, and $9 \mathrm{~mL}$ of the PIF mixture was inoculated with $1 \mathrm{~mL}$ of above-mentioned 10-fold dilution of C. sakazakii culture, respectively. The PIF contaminated by C. sakazakii ATCC 29544 underwent the processes of IMS, DNA extraction, and LAMP assay; meanwhile, the uncontaminated PIF served as a negative control.

\section{Process of IMS, DNA Extraction, and LAMP Assay for Cronobacter spp. in PIF with a Portable Electricity-Free Heater}

Process of IMS. The weight of PCM $\left(35^{\circ} \mathrm{C}\right)$ that was added to the upper chamber was fixed at $8 \mathrm{~g}$, and the proportion of calcium oxide and water was optimized within the trial and repeated 3 times. Then, the mixture of 2 reagents was introduced into the bottom chamber by stirring. Meanwhile, $400 \mu \mathrm{L}$ of PIF contaminated by different concentrations of bacteria was blended with $50 \mu \mathrm{L}$ of MNB-Ab in a 2-mL epoxy epoxide tube. Then the epoxy epoxide tube was put into PCM through the hole to capture strains by IMS, and was incubated for $1 \mathrm{~h}$ at $35^{\circ} \mathrm{C}$ with a portable electricity-free heater. Next, after magnetic separation for $2 \mathrm{~min}$, the supernatant was removed and mixtures were washed 3 times by PBS solution. After magnetic separation, the supernatant was removed; then, the captured bacteria strains were resuspended in $100 \mu \mathrm{L}$ of PBS solution and placed on a magnet for $1 \mathrm{~min}$. The total process was repeated 4 times. The first time was conducted to remove supernatant to prepare DNA extraction, and the other 3 times were to conducted to calculate the concentration of bacteria in PIF by the plate counting method.

Process of DNA Extraction. The weight of PCM $\left(100^{\circ} \mathrm{C}\right)$, which was added to the upper chamber, fixed at $9 \mathrm{~g}$. The optimal ratio of calcium oxide and water was determined by repeating 3 times; then, the mixture of 2 materials was added to the bottom chamber by stirring. The above-mentioned captured bacteria in a 2-mL epoxy epoxide tube with different concentrations were mixed with $30 \mu \mathrm{L}$ of DNA lysis buffer $[\mathrm{pH} 8.0$, $10 \mathrm{~m} M$ Tris, $4.5 \%$ (vol/vol) Triton X-100, $4.5 \%$ (vol/ vol) Tween 20], respectively, then the epoxy epoxide tube was embedded into PCM through the hole and boiled for $3 \mathrm{~min}$ at $100^{\circ} \mathrm{C}$ to complete DNA extraction with the portable electricity-free heater. Finally, the mixtures as DNA template were injected into the LAMP reaction.

Process of LAMP Assay. The weight of PCM $\left(65^{\circ} \mathrm{C}\right)$ that was added to the upper chamber fixed at $10 \mathrm{~g}$ as an invariant, and the proportion of calcium oxide and water was tested to obtain the optimal ratio. Two reagents were added to the bottom chamber by stirring, repeating the optimization experiment 3 times. The LAMP mixtures in the $200-\mu \mathrm{L}$ PCR tube were inserted into PCM; then, the LAMP reaction was 


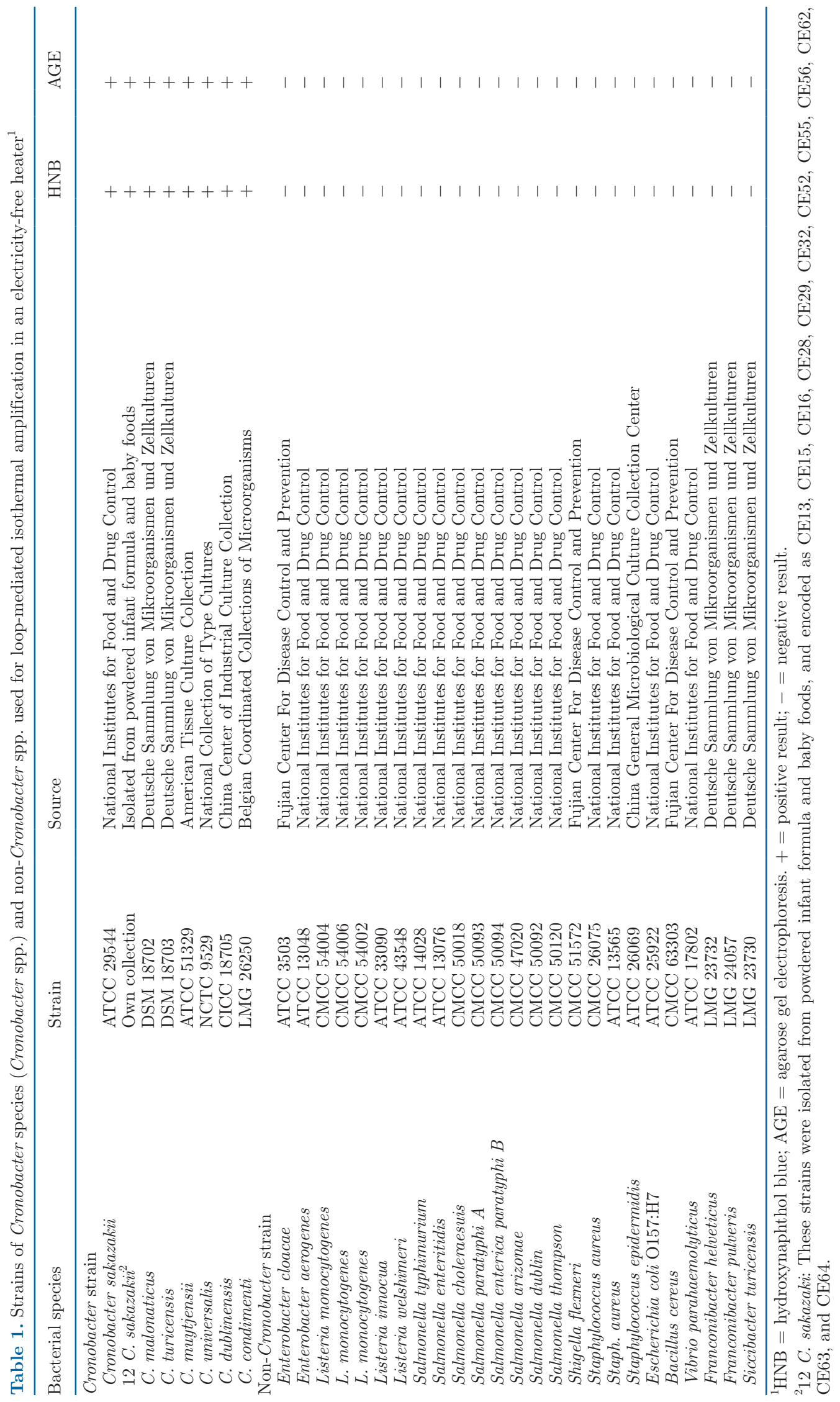


performed with a portable electricity-free heater. In this investigation, LAMP primer sequences targeting the internal transcribed spacer (ITS) of Cronobacter spp. were described previously, which including 2 outer primers (F3 and B3), 2 inner primers (FIP and BIP), and 2 loop primers (LF and LB; Liu et al., 2009). The optimal conditions of the LAMP assay were determined by the portable electricity-free heater, including reaction time and temperature, proportion of primers, and the concentration of reagents. The reagents consisted of dNTP, Bst 2.0 WarmStart DNA polymerase (New England BioLabs, Ipswich, MA), and $\mathrm{MgSO}_{4}$.

Visual Detection of Amplification Products. The LAMP products were visually detected by color changing of $\mathrm{HNB}$, and a positive result with color of sky blue was observed following the addition of HNB in LAMP reaction beforehand, whereas a negative result showed violet. To prove the reliability of this method, $2 \%$ agarose gel electrophoresis (AGE) and optical density (OD) detection was conducted. The results were valued according to the electrophoretic band using AGE and the ratio of OD value tested in different wavelengths of light by a microplate reader.

\section{Specificity and Sensitivity Test of LAMP for Cronobacter spp.}

Specificity of LAMP Assay for Cronobacter spp. The DNA from 19 Cronobacter spp. strains and 25 non-Cronobacter spp. strains were used to determine the specificity of the LAMP assay. The LAMP reaction was conducted in a portable electricity-free heater. The results were observed by color changing of HNB and electrophoretic band under UV light after AGE.

Sensitivity for Cronobacter spp. in Powdered Infant Formula. The IMS, DNA extraction, and LAMP assay were performed by using a portable electricity-free heater and conventional electric device, respectively. The above-mentioned DNA extracted from different concentrations of C. sakazakii ATCC 29544 in PIF served as positive templates, and sterile distilled water was used as a negative control to carry out the LAMP assay, and the amplified products were detected by $\mathrm{HNB}$ and $2 \%$ AGE to determine the sensitivity. At the same time, the traditional PCR method was carried out, and LAMP outer primers (F3 and B3) were used as PCR primers to compare the detection sensitivity.

\section{Interference and Stability Test for LAMP with Portable Electricity-Free Heater}

To test the interference of background microflora in the PIF samples, Listeria monocytogenes CMCC 54004, Salmonella typhimurium ATCC 14028, Escherichia coli
O157:H7 ATCC 25922, and Bacillus cereus CMCC 63303 were cultured to achieve different concentrations of strains by serial 10-fold dilution. Next, the above non-Cronobacter spp. were mixed with C. sakazakii in equal volumes to obtain contaminated PIF. The final concentrations of non-Cronobacter spp. ranged from $10^{\circ}$ to $10^{8} \mathrm{cfu} / \mathrm{g}$, whereas that of C. sakazakii fixed at $10^{7}$ $\mathrm{cfu} / \mathrm{g}$. Then DNA was extracted from the mixtures and was used as a template for LAMP-HNB and LAMPAGE detection with a portable electricity-free heater. Additionally, the stability of the heater and detection limit was tested with electricity-free amplification and visual detection system at $4^{\circ} \mathrm{C}, 25^{\circ} \mathrm{C}$, and $37^{\circ} \mathrm{C}$, respectively.

\section{RESULTS AND DISCUSSION}

\section{Optimization of IMS, DNA Extraction, and LAMP Assay}

Process of IMS. The components of PIF were complicated, which was unfavorable for DNA extraction, so pre-concentration and purification for targeted bacteria were necessary. Even though IMS have been applied to concentrate the pathogenic bacteria, a previous study only conducted IMS by utilizing conventional electric heaters, such as a shaking table and incubator (Yang et al., 2013). In this assay, an electricity-free heater was introduced to perform IMS, and the weight of PCM $\left(35^{\circ} \mathrm{C}\right)$ and calcium oxide fixed at 8 and $15 \mathrm{~g}$, and the volume of water was added at 1.4, 1.6, 1.8, and $2.0 \mathrm{~mL}$, respectively. The temperature profile of the electricityfree heater was generated by calculating the mean and standard deviation of temperature (Figure 2). The results indicated that $15 \mathrm{~g}$ of calcium oxide and 1.6 $\mathrm{mL}$ of water combining with $8 \mathrm{~g}$ of PCM $\left(35^{\circ} \mathrm{C}\right)$ could maintain $60 \mathrm{~min}$ at $35^{\circ} \mathrm{C}$ to capture the Cronobacter spp. by IMS. At this temperature, the MNB-Ab was used to capture the bacteria to form conjugate that can be separated by a magnet. A $2-\mathrm{mL}$ epoxy epoxide tube was taken out from heater at 67 min including warm-up time. Therefore, the method was more appropriate for resource-limited settings compared with the previous method, and electricity and complicated instruments were not required.

Process of DNA Extraction. To detect the captured bacteria, DNA was needed as template to carry out nucleic acid amplification, and various DNA extraction methods were reported (Wu et al., 2014; Bletz et al., 2015). However, the DNA kit was expensive, time consuming, and complicated. The boiling method was low cost and rapid, but centrifuge was needed. The phenol-chloroform isoamyl alcohol method and glass bead method needed steps of centrifugation and vor- 
tex. Hence, these techniques were not appropriate for on-site detection. In this assay, DNA lysis buffer was added to the electricity-free heater to solve the above problems. The weights of $\mathrm{PCM}\left(100^{\circ} \mathrm{C}\right)$ and calcium oxide were fixed at 9 and $42 \mathrm{~g}$, whereas the volume of water was added at $5,6,7,7.5 \mathrm{~mL}$, respectively. The temperature profile of electricity-free heater was plotted by calculating the mean and standard deviation of temperature (Figure 3). The results showed that $42 \mathrm{~g}$ of calcium oxide and $7 \mathrm{~mL}$ of water together with $9 \mathrm{~g}$ of PCM $\left(100^{\circ} \mathrm{C}\right)$ could maintain 30 min at $100^{\circ} \mathrm{C}$, which aimed to extract DNA of Cronobacter spp. by adding the lysis buffer method. A 2-mL epoxy epoxide tube was removed from the heater after 15 min including warm-up time; thus, the temperature profile suggested that a portable electricity-free heater could complete the DNA extraction. The method was simple, rapid, and low cost, and did not need an electric device; meanwhile, the process of IMS and DNA extraction could be completed with a portable electricity-free heater in $1 \mathrm{~h}$ $30 \mathrm{~min}$.

Process of LAMP Assay. The weights of PCM $\left(65^{\circ} \mathrm{C}\right)$ and calcium oxide were fixed at 10 and $30 \mathrm{~g}$, respectively, and the volume of water was added at 4, 5,6 , and $7 \mathrm{~mL}$. The temperature profile was generated similarly as above. Figure 4 illustrated that $30 \mathrm{~g}$ of calcium oxide and $5 \mathrm{~mL}$ of water combined with $10 \mathrm{~g}$ of PCM $\left(65^{\circ} \mathrm{C}\right)$ could keep $45 \mathrm{~min}$ at $65^{\circ} \mathrm{C}$ for the LAMP assay, so the $200-\mu \mathrm{L}$ PCR tube was taken out from the heater approximately at 60 min including preheating time. After optimization of LAMP reaction with the portable electricity-free heater, the optimal LAMP assay was performed in $25 \mu \mathrm{L}$ of a mixture containing $2 \mu \mathrm{L}$ of extracted DNA template after IMS, 1.6

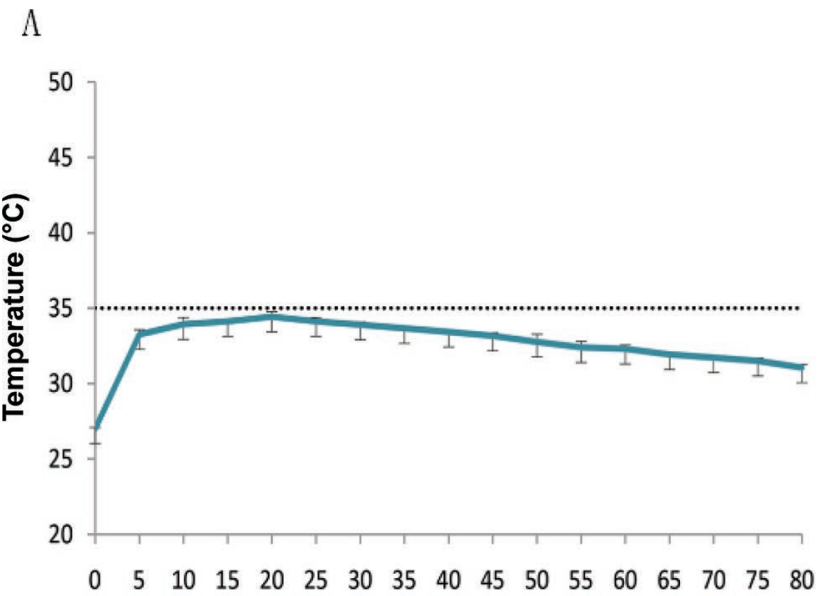

$$
\text { B }
$$
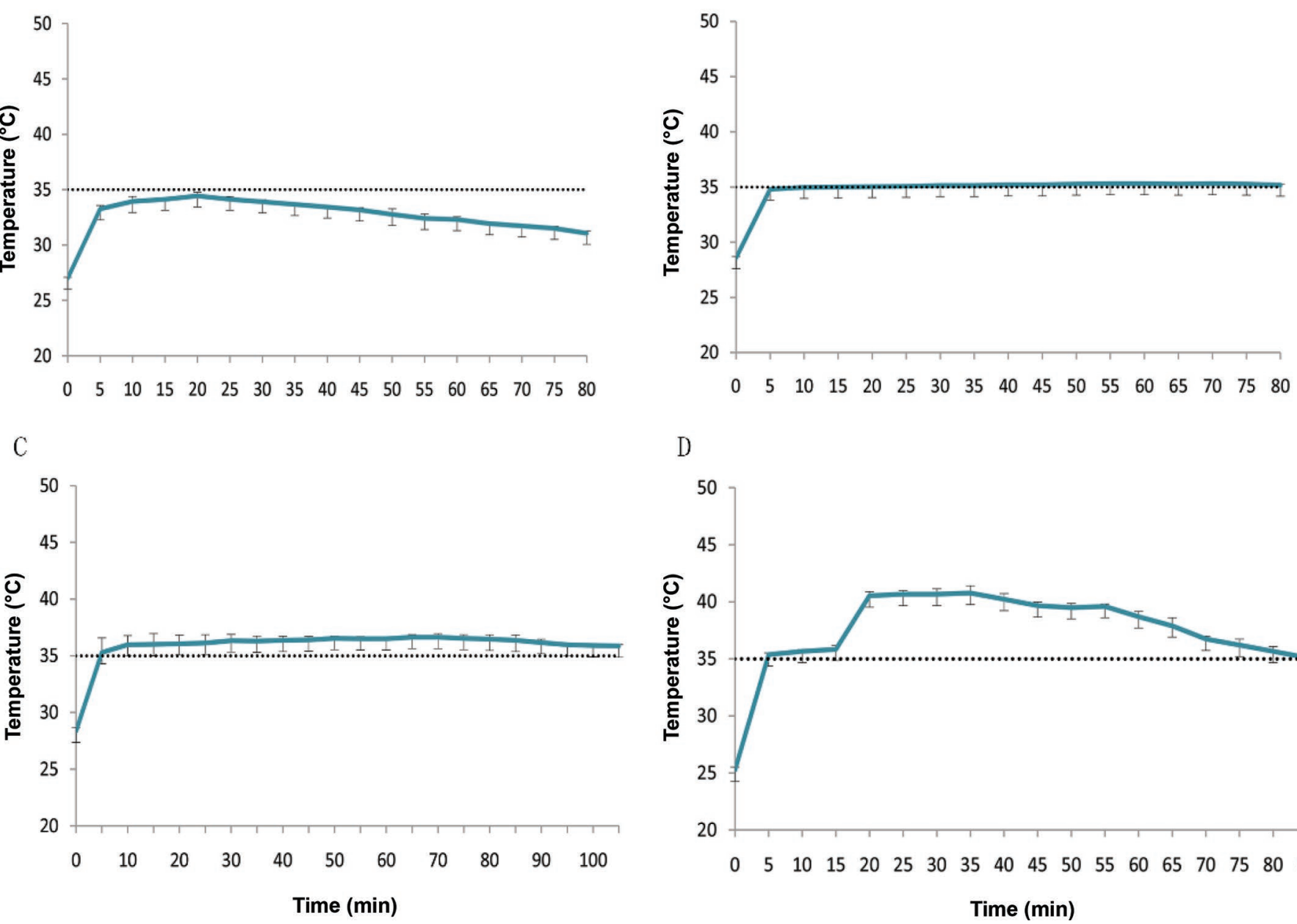

D

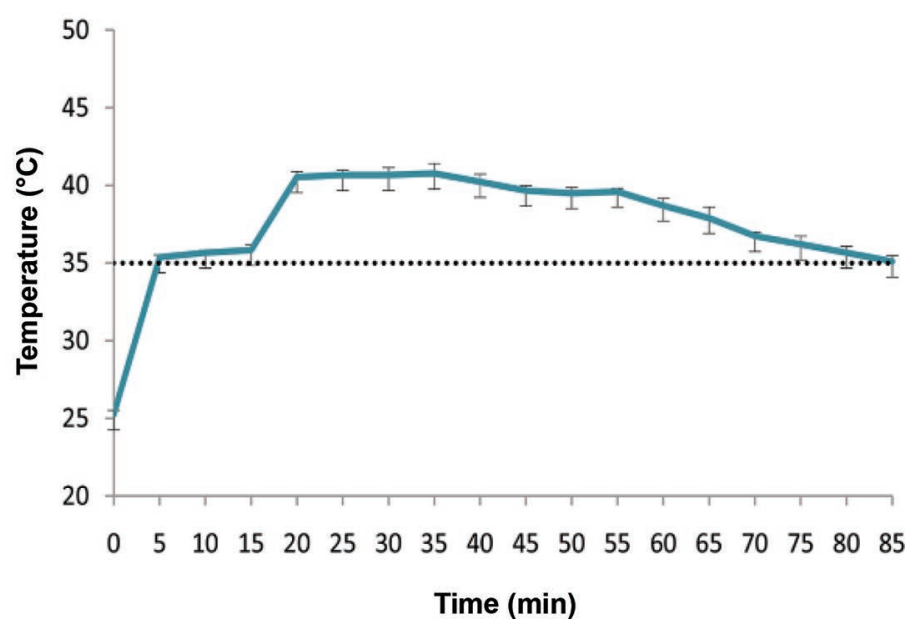

Figure 2. The optimization of the proportion of calcium oxide and water combining with phase change material $\left(\mathrm{PCM}, 35^{\circ} \mathrm{C}\right)$. The weights of PCM and calcium oxide were 8 and $15 \mathrm{~g}$, respectively. A-D: the volume of water was 1.4, 1.6, 1.8, and $2.0 \mathrm{~mL}$, respectively. Data shown as mean $\pm \mathrm{SD}, \mathrm{n}=3$. 

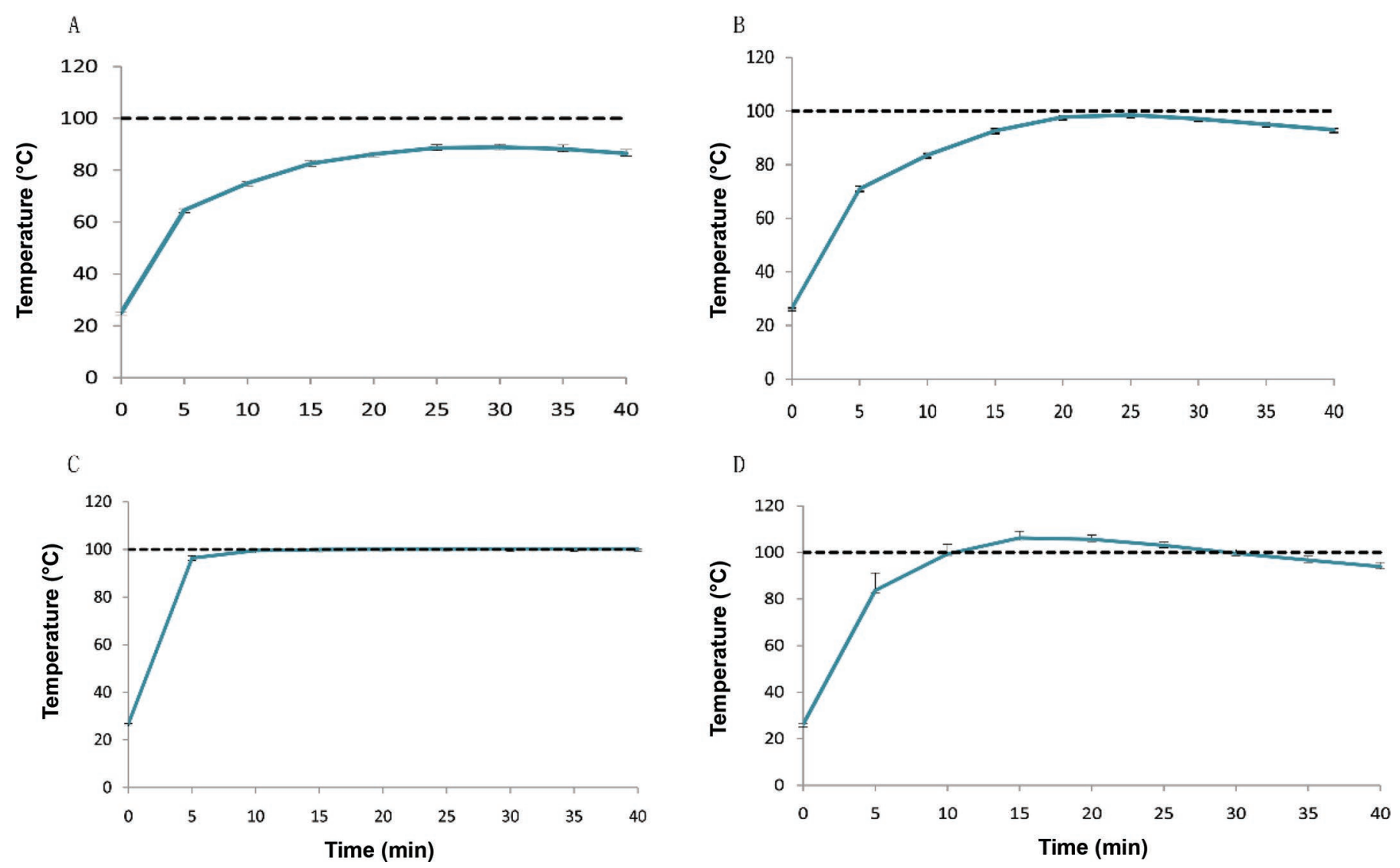

Figure 3. The optimization of the proportion of calcium oxide and water combining with phase change material $\left(\mathrm{PCM}, 100^{\circ} \mathrm{C}\right)$. The weights of PCM and calcium oxide were 9 and $42 \mathrm{~g}$, respectively. A-D: the volume of water was 5, 6, 7, and $7.5 \mathrm{~mL}$, respectively. Data shown as mean $\pm \mathrm{SD}, \mathrm{n}=3$.

$\mu M$ each of the FIP and BIP primers, $0.2 \mu M$ each of the F3 and B3 primers, $0.8 \mu M$ each of the LF and LB primers, and $0.8 \mu \mathrm{L}$ of Bst 2.0 WarmStart DNA polymerase $(8 \mathrm{U} / \mu \mathrm{L}), 2.5 \mu \mathrm{L}$ of $10 \times$ ThermoPol Buffer $\left(200 \mathrm{~m} M\right.$ Tris-HCl, $500 \mathrm{mM} \mathrm{KCl,} 20 \mathrm{mM} \mathrm{MgSO}_{4}$, $100 \mathrm{mM}(\mathrm{NH} 4)_{2} \mathrm{SO}_{4}, 1 \%$ Tween $20(\mathrm{pH} 8.8)$ at $\left.25^{\circ} \mathrm{C}\right)$, $2 \mathrm{mM}$ dNTP, $4 \mathrm{mM} \mathrm{MgSO}_{4}, 4.2 \mu \mathrm{L}$ of double-distilled $\mathrm{H}_{2} \mathrm{O}$, and $150 \mu M \mathrm{HNB}$, which could attain the visual detection of Cronobacter spp. in PIF. Subsequently, the system was performed at $65^{\circ} \mathrm{C}$ for $45 \mathrm{~min}$. The LAMP assay combined with an electricity-free heater did not require skilled personnel and costly equipment; thus, it could be completed under limited conditions.

Among nucleic acid amplification detection, the LAMP reaction only required a constant temperature, and various visualized detection methods for LAMP products have been developed, such as lateral flow dipsticks, Quant-iT PicoGreen, and SYBR Green I (Wang et al., 2015; Syedmoradi et al., 2017). However, the opening reaction tube exposed in air made the products be easily contaminated and gave rise to false-positive results. The calcein and $\mathrm{MnCl}_{2}$ assay showed a series of partial inhibition and color changing was difficult to observe. Our HNB detection method enabled instant visualization of LAMP products without complicated equipment needing electricity. Additionally, HNB was introduced into the LAMP reaction beforehand; thus, it could avoid cross-contamination of LAMP reagents and amplification products.

\section{Establishment of the Electricity-Free Amplification and Visual Detection System}

Although the methods of IMS, DNA isolation, and LAMP assay have been developed to detect pathogens (Sun et al., 2015), they needed to be accomplished in electricity-settings or laboratories. The previous study also revealed that the LAMP assay was hardly conducted by electricity-free heaters in limited conditions (Kubota et al., 2011). In this investigation, the IMS, DNA extraction, and LAMP assay with a portable electricity-free heater could maintain the required temperature and time as described above. Although the reaction process could be conducted, the visual detection 

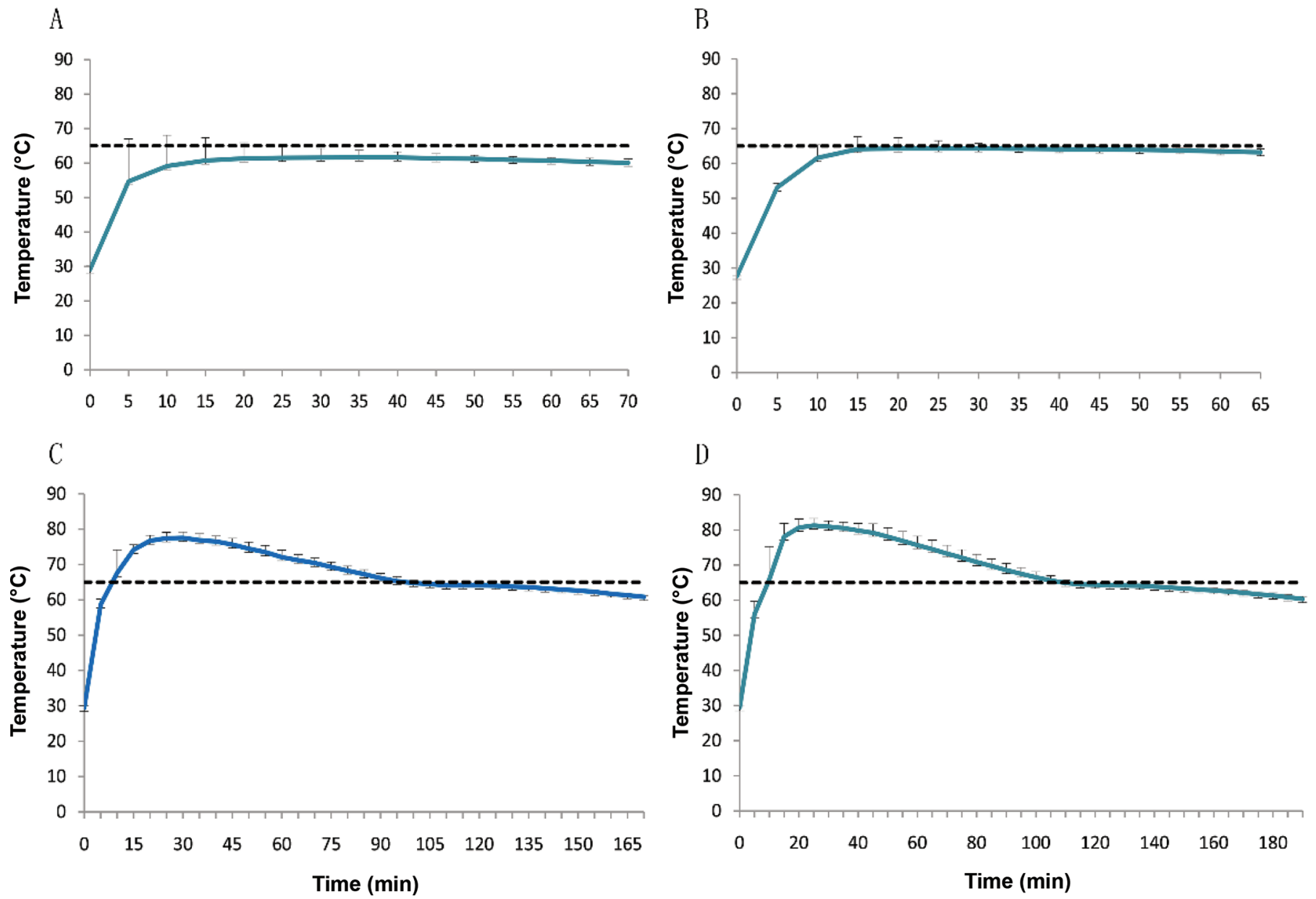

Figure 4. The optimization of the proportion of calcium oxide and water combining with phase change material $\left(\mathrm{PCM}, 65^{\circ} \mathrm{C}\right)$. The weights of PCM and calcium oxide were 10 and $30 \mathrm{~g}$, respectively. A-D: the volume of water was 4, 5, 6 , and $7 \mathrm{~mL}$, respectively. Data shown as mean $\pm \mathrm{SD}, \mathrm{n}=3$.

was not solved. Thus, to further prove the capacity for detection, our HNB detection method was introduced to realize instant visualization of LAMP products without specialized equipment, and the LAMP assay could not be terminated. Meanwhile, the process of IMS, DNA extraction, and LAMP-HNB assay was performed using an electricity-free heater and conventional electric device, respectively. Then, the LAMP products obtained by two devices were observed by AGE under UV light (Figure 5A) and color of HNB (Figure 5B). The results of AGE demonstrated that an electricityfree heater can supply the same reaction conditions as a conventional electric heater, and the LAMP reaction in a portable electricity-free heater produced a pattern of electrophoresis similar to that of a conventional electric heater. Meanwhile, the results of LAMP products by HNB method (via color changing) were in accordance with the AGE assay, which further illustrated the favorable stability of the electricity-free heater. At the same time, the ratio of $\mathrm{OD}_{500 \mathrm{~nm}}$ and $\mathrm{OD}_{650 \mathrm{~nm}}$ were tested to verify the rationality of the visual detection method (Supplemental Table S1; https://doi.org/10.3168/jds .2019-17661). The results also showed a significant difference between positive samples from 2 devices and negative control. Thus, the feasibility of the visual detection system was confirmed by detecting the Cronobacter spp., and the total detection process did not require expensive instruments and skilled operators. Furthermore, the total visual detection time containing IMS, DNA extraction, and LAMP-HNB assay can be controlled in $2 \mathrm{~h} 30$ min without preincubation steps of bacteria.

\section{Specificity of LAMP Assay with a Portable Electricity-Free Heater}

Our heater as heat source needed warm-up at lower reaction temperature to implement the LAMP assay 
A

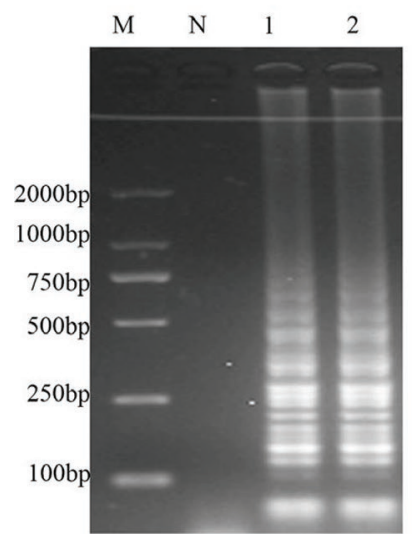

Figure 5. The results of detection of Cronobacter spp. by conducting immunomagnetic separation, DNA extraction, and loop-mediated isothermal amplification in the different devices. (A) Agarose gel electrophoresis. (B) Hydroxynaphthol blue. M: marker; lane 1: portable electricity-free heater; lane 2: conventional electric heater; $\mathrm{N}$ : negative control.

and the LAMP primers were designed in a previous screening. To test if the LAMP assay with the electricity-free heater could produce specific amplification and specificity of LAMP primers, the LAMP products were observed by changing color of HNB and AGE under UV light, respectively. The results demonstrated that all 19 Cronobacter spp. strains showed a sky blue color and all 25 non-Cronobacter spp. strains were violet by the HNB method with a portable electricity-free heater as shown in Table 1. Meanwhile, the positive reaction was seen as a ladder-like pattern on 2\% AGE analysis, which contained all 19 Cronobacter spp. strains, whereas no band of the negative reaction contained all 25 non-Cronobacter spp. strains.

The sequences of positive amplified products were measured by utilizing DNA sequencing technology, which illustrating the consistency with targeted fragments in ITS. At the same time, LAMP primers were proven to be specific to the selected region and used to identify Cronobacter spp. with our electricity-free amplification detection system. Similar results appeared in previous reports. Liu et al. (2012) used ITS sequence of Cronobacter spp. to design LAMP primers for rapidly detecting Cronobacter spp., and no false-positive amplification was observed in the 14 non-Cronobacter spp. microorganisms. Fu et al. (2018) developed a LAMPLFD method based on the ITS sequence for visual detection of Cronobacter spp., which showed positive results of 19 Cronobacter spp. strains and negative results of all 29 strains of non-Cronobacter. These further illustrated the reliability of the 16S-23S ITS sequence to distinguish Cronobacter spp. from all other bacteria.

\section{Sensitivity for Cronobacter spp. in Artificially Contaminated PIF}

To test the sensitivity of our electricity-free amplification and visual detection system, C. sakazakii ATCC 29544 was chosen as the target bacterium for performing IMS, DNA extraction, and LAMP assay. Meanwhile, the sensitivity was determined to compare with a conventional electric device and traditional PCR method by amplifying target bacteria in PIF at known concentrations. In this paper, the sensitivities for PIF contaminated with $C$. sakazakii using a conventional electric device and electricity-free heater manifested as shown in Figure 6. The results of HNB and AGE illustrated that the detection limit of the contaminated Cronobacter spp. in PIF was $3.8 \times 10^{2} \mathrm{cfu} / \mathrm{g}$ in electric devices by using 2 detection methods. The detection limit was $4.2 \times 10^{2} \mathrm{cfu} / \mathrm{g}$ in an electricity-free heater, whereas it was $2.6 \times 10^{3} \mathrm{cfu} / \mathrm{g}$ with the PCR method, indicating no significant difference by utilizing an electricity-free heater and conventional electric devices, and the LAMP-HNB method established in this investigation had the same sensitivity as the LAMP-AGE, which was 10 times that of the traditional PCR method. In previous reports, Chen et al. (2017) developed the IMS-PCR method to detect Cronobacter spp. and the sensitivity was $5.2 \times 10^{2} \mathrm{cfu} / \mathrm{mL}$ in artificially contaminated PIF without enrichment. The detection limit in milk was $2.8 \times 10^{4} \mathrm{cfu} / \mathrm{mL}$ by utilizing a noninstrumented platform (Kubota et al., 2013). Therefore, the established system was certified to have high sensitivity. To further realize the applicability for real samples, we found the established system could detect Cronobacter spp. with a level of $10^{\circ} \mathrm{cfu} / \mathrm{g}$ in artificially contaminated PIF after $2 \mathrm{~h}$ of cultivation (Supplemental Table S2; https://doi.org/10.3168/jds .2019-17661).

\section{Interference Test for LAMP Using Various Concentrations of Non-Cronobacter spp.}

There generally existed background microflora in PIF in addition to the Cronobacter spp., which could affect the LAMP reaction to cause false-positive or false-negative results. Hence, a mixture of DNA from $C$. sakazakii and 4 non-Cronobacter spp. in PIF was used to evaluate LAMP interference by our electricity-free heater. As shown in Figure 7, the sky blue color of HNB and trapezoidal bands of AGE appeared with bacterial concentrations from $10^{2}$ to $10^{8} \mathrm{cfu} / \mathrm{g}$, whereas the violet color and no bands appeared when the bacterial concentrations were less than $10^{2} \mathrm{cfu} / \mathrm{g}$. The results were consistent with the specificity and sensitivity detection of Cronobacter spp., indicating that our electricity-free 
A

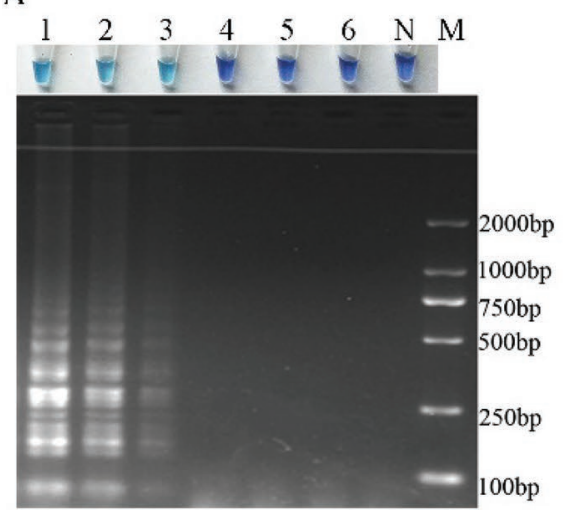

B

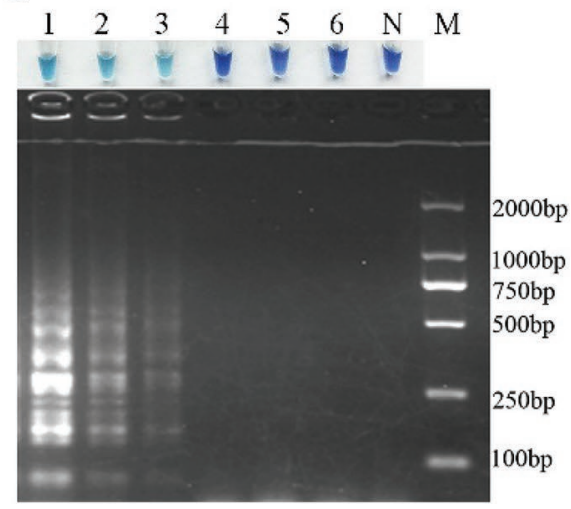

$\mathrm{C}$

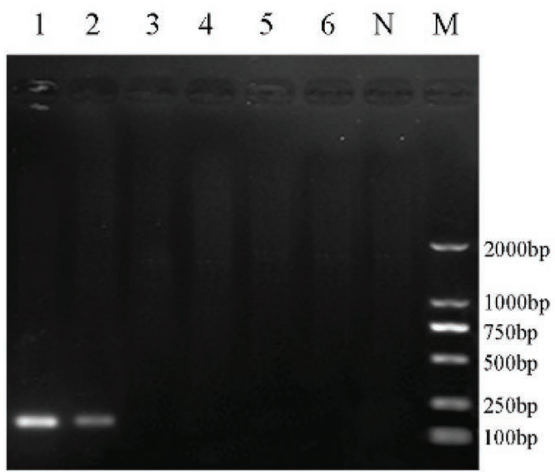

Figure 6. The sensitivity for powdered infant formula (PIF) contaminated with Cronobacter spp. using different devices and methods. (A) Conventional electric heater. Lanes 1 to 6: the PIF contaminated with concentrations of Cronobacter sakazakii ATCC 29544 ranging from 3.8 $\times 10^{4}$ to $3.8 \times 10^{-1} \mathrm{cfu} / \mathrm{g}$, respectively. (B) Electricity-free heater. Lanes 1 to 6 : the PIF contaminated with concentrations of $C$. sakazakii ATCC 29544 ranging from $4.2 \times 10^{4}$ to $4.2 \times 10^{-1} \mathrm{cfu} / \mathrm{g}$, respectively. Loop-mediated isothermal amplification products were examined by hydroxynaphthol blue (upper lane) and agarose gel electrophoresis (lower lane). (C) Traditional PCR method. Lanes 1 to 6: the PIF contaminated with concentrations of C. sakazakii ATCC 29544 ranging from $2.6 \times 10^{4}$ to $2.6 \times 10^{-1} \mathrm{cfu} / \mathrm{g}$, respectively. M: marker; N: negative control.

amplification and visual detection system was not affected by DNA from other bacteria.

\section{Stability Assay in Different Ambient Temperatures}

Because the detection process was conducted in limited-resource settings, the equipment may suffer from high or low temperatures. Therefore, the stability of an electricity-free heater may have a fluctuant result of

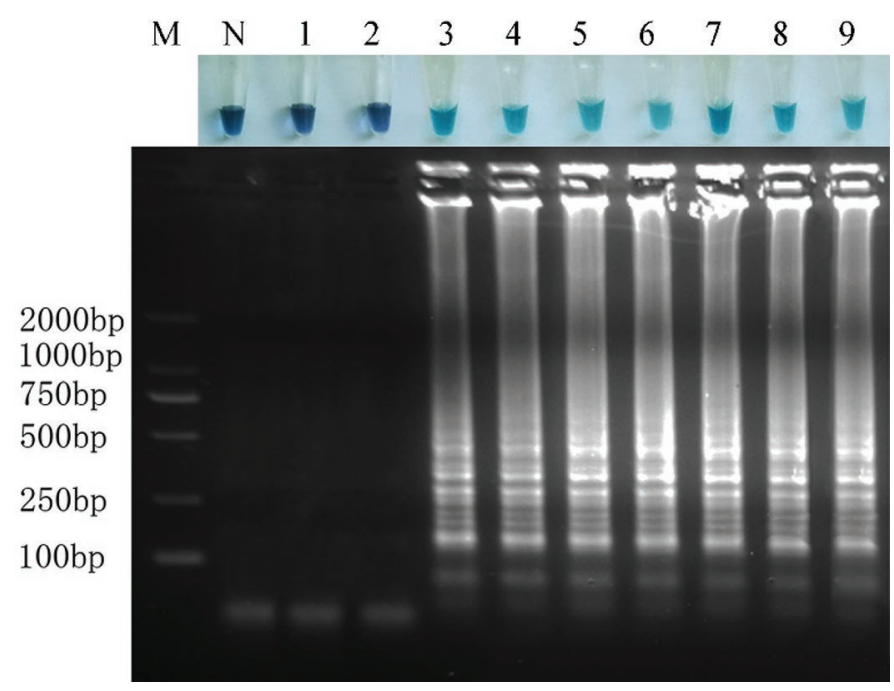

Figure 7. Interference test for Cronobacter spp. with different concentrations of non-Cronobacter spp. M: marker; N: negative control; lanes 1-9: the powdered infant formula contaminated with Cronobacter sakazakii ATCC 29544 (concentration fixed at $10^{7} \mathrm{cfu} / \mathrm{g}$ ) and 4 non-Cronobacter spp. ranging from $10^{0}$ to $10^{8} \mathrm{cfu} / \mathrm{g}$, respectively. Loop-mediated isothermal amplification products were examined by hydroxynaphthol blue (upper lane) and agarose gel electrophoresis (lower lane). unstable sensitivity of PIF contaminated with Cronobacter spp. To certify the stability and the reliability of detection limit of the electricity-free heater, the experiment was carried out at 3 representative temperatures of $4^{\circ} \mathrm{C}, 25^{\circ} \mathrm{C}$ and $37^{\circ} \mathrm{C}$ by using an electricity-free heater, and the products were detected by HNB. The results suggested that a stable reaction temperature could be maintained, whereas the sensitivity had no significant difference and could attain $10^{2} \mathrm{cfu} / \mathrm{g}$ (Table 2 ). Consequently, the stability and sensitivity were reliable in ambient temperatures of operation ranging from $4^{\circ} \mathrm{C}$ to $37^{\circ} \mathrm{C}$.

\section{CONCLUSIONS}

We designed a portable electricity-free heater that integrated the processes of IMS, DNA extraction, and LAMP assay of PIF contaminated with Cronobacter spp. It is a simple, low cost, chemically heated, and portable device without electrical power. At the same time, HNB was added to the LAMP reaction in advance to achieve visual detection of amplified products and avoid cross-contamination, high cost, and utilization of electrical instruments. The visual detection system we established showed high specificity for Cronobacter

Table 2. The detection limit of the Cronobacter spp. in powdered infant formula using a portable electricity-free heater at different ambient temperatures

\begin{tabular}{lccc}
\hline & \multicolumn{3}{c}{ Ambient temperature $\left({ }^{\circ} \mathrm{C}\right)$} \\
\cline { 2 - 4 } Item & 4 & 25 & 37 \\
\hline Detection limit $(\mathrm{cfu} / \mathrm{g})$ & $3.6 \times 10^{2}$ & $4.5 \times 10^{2}$ & $4.7 \times 10^{2}$ \\
\hline
\end{tabular}


spp. and the sensitivity can reach $10^{2} \mathrm{cfu} / \mathrm{g}$ in PIF, even at different ambient temperatures. The total detection time was less than $2 \mathrm{~h} 30 \mathrm{~min}$. Furthermore, Cronobacter spp. can be detected at a concentration of $10^{0} \mathrm{cfu} / \mathrm{g}$ in artificially contaminated PIF after $2 \mathrm{~h}$ of pre-enrichment. In short, compared with conventional LAMP assays for Cronobacter spp., this LAMP-HNB visual method with a portable electricity-free heater will provide a rapid, simple, specific, and sensitive detection system for Cronobacter spp. in PIF in limited-resource settings, and can be applied to other food products.

\section{ACKNOWLEDGMENTS}

This study was supported by National Natural Science Foundation of China (no. 31871828), National High-Level Talents Special Support Program of China, and National Key Research and Development Program of China (no. 2018YFE0120500). We declare that we do not have any commercial or associative interest that represents a conflict of interest in connection with the work submitted.

\section{REFERENCES}

Abdelhai, M. H., H. A. M. Hassanin, and X. Sun. 2016. Comparative study of rapid DNA extraction methods of pathogenic bacteria. J. Biosci. Bioeng. 4:1-8. https://doi.org/10.11648/j.bio.20160401.11.

Bletz, M. C., E. A. Rebollar, and R. N. Harris. 2015. Differential efficiency among DNA extraction methods influences detection of the amphibian pathogen Batrachochytrium dendrobatidis. Dis. Aquat. Organ. 113:1-8. https://doi.org/10.3354/dao02822.

Chen, Q., Y. Li, T. Tao, X. Bie, F. Lu, and Z. Lu. 2017. Development and application of a sensitive, rapid, and reliable immunomagnetic separation-PCR detection method for Cronobacter spp. J. Dairy Sci. 100:961-969. https://doi.org/10.3168/jds.2016-11087.

Curtis, K. A., D. L. Rudolph, I. Nejad, J. Singleton, A. Beddoe, B. Weigl, P. Labarre, and S. M. Owen. 2012. Isothermal amplification using a chemical heating device for point-of-care detection of HIV-1. PLoS One 7:e31432. https://doi.org/10.1371/journal.pone .0031432 .

Fu, S., Y. Jiang, X. Jiang, Y. Zhao, S. Chen, X. Yang, and C. Man. 2018. Probe-free label system for rapid detection of Cronobacter genus in powdered infant formula. AMB Express 8:155. https:// doi.org/10.1186/s13568-018-0689-x.

Hatano, B., T. Maki, T. Obara, H. Fukumoto, K. Hagisawa, Y. Matsushita, A. Okutani, B. Bazartseren, S. Inoue, and T. Sata. 2010. LAMP using a disposable pocket warmer for anthrax detection, a highly mobile and reliable method for anti-bioterrorism. Jpn. J. Infect. Dis. 63:36-40. https://doi.org/10.1258/ijsa.2009.008500.

Healy, B., S. Cooney, S. O'Brien, C. Iversen, P. Whyte, J. Nally, J. J. Callanan, and S. Fanning. 2010. Cronobacter (Enterobacter sakazakii): An opportunistic foodborne pathogen. Foodborne Pathog. Dis. 7:339-350. https://doi.org/10.1089/fpd.2009.0379.

Huang, X., Z. P. Aguilar, H. Xu, W. Lai, and Y. Xiong. 2016. Membrane-based lateral flow immunochromatographic strip with nanoparticles as reporters for detection: A review. Biosens. Bioelectron. 75:166-180. https://doi.org/10.1016/j.bios.2015.08.032.

Kim, H. S., Y. J. Kim, J. W. Chon, D. H. Kim, and K. H. Seo. 2016. Two-stage label-free aptasensing platform for rapid detection of Cronobacter sakazakii in powdered infant formula. Sensor. Actuat. Biol. Chem. 239:94-99. https://doi.org/10.1016/j.snb.2016.07.173.
Kubota, R., P. Labarre, J. Singleton, A. Beddoe, B. H. Weigl, A. M. Alvarez, and D. M. Jenkins. 2011. Non-instrumented nucleic acid amplification (NINA) for rapid detection of Ralstonia solanacearum Race 3 biovar 2. Biol. Eng. Trans. 4:69-80. https://doi .org/10.13031/2013.38508.

Kubota, R., P. Labarre, B. H. Weigl, Y. Li, P. Haydock, and D. M. Jenkins. 2013. Molecular diagnostics in a teacup: Non-instrumented nucleic acid amplification (NINA) for rapid, low cost detection of Salmonella enterica. Chin. Sci. Bull. 58:1162-1168. https://doi .org/10.1007/s11434-012-5634-9.

Labarre, P., J. Gerlach, J. Wilmoth, A. Beddoe, J. Singleton, and B. Weigl. 2010. Non-instrumented nucleic acid amplification (NINA): Instrument-free molecular malaria diagnostics for low-resource settings. Conf. Proc. IEEE Eng. Med. Biol. Soc. 2010:1097-1099. https://doi.org/10.1109/IEMBS.2010.5627346.

Labarre, P., K. R. Hawkins, J. Gerlach, J. Wilmoth, A. Beddoe, J. Singleton, D. Boyle, and B. Weigl. 2011. A simple, inexpensive device for nucleic acid amplification without electricity-toward instrument-free molecular diagnostics in low-resource settings. PLoS One 6:e19738. https://doi.org/10.1371/journal.pone.0019738.

Liao, S. C., J. Peng, M. G. Mauk, S. Awasthi, J. Song, H. Friedman, H. H. Bau, and C. Liu. 2016. Smart Cup: A minimally-instrumented, smartphone-based point-of-care molecular diagnostic device. Sens. Actuators B Chem. 229:232-238. https://doi.org/10.1016/ j.snb.2016.01.073.

Liu, C., M. G. Mauk, R. Hart, X. Qiu, and H. H. Bau. 2011. A selfheating cartridge for molecular diagnostics. Lab Chip 11:2686. https://doi.org/10.1039/c1lc20345b.

Liu, C., W. J. Zheng, H. W. Zhang, Y. M. Hou, and Y. Liu. 2009. Sensitive and rapid detection of Enterobacter sakazakii in infant formula by loop-mediated isothermal amplification method. J. Food Saf. 29:83-94. https://doi.org/10.1111/j.1745-4565.2008.00142.x.

Liu, X., J. Fang, M. Zhang, X. Wang, W. Wang, Y. Gong, X. Xi, and M. Li. 2012. Development of a loop-mediated isothermal amplification assay for detection of Cronobacter spp. (Enterobacter sakazakii). World J. Microbiol. Biotechnol. 28:1013-1020. https:// doi.org/10.1007/s11274-011-0899-8.

Luo, K., L. Hu, Q. Guo, C. Wu, S. Wu, D. Liu, Y. Xiong, and W. Lai. 2017. Comparison of 4 label-based immunochromatographic assays for the detection of Escherichia coli O157:H7 in milk. J. Dairy Sci. 100:5176-5187. https://doi.org/10.3168/jds.2017-12554.

Norberg, S., C. Stanton, R. P. Ross, C. Hill, G. F. Fitzgerald, and P. D. Cotter. 2012. Cronobacter spp. in powdered infant formula. J. Food Prot. 75:607-620. https://doi.org/10.4315/0362-028X.JFP $-11-285$.

Pecchia, S., and D. Da Lio. 2018. Development of a rapid PCR-nucleic acid lateral flow immunoassay (PCR-NALFIA) based on rDNA $I G S$ sequence analysis for the detection of Macrophomina phaseolina in soil. J. Microbiol. Methods 151:118-128. https://doi.org/10 .1016/j.mimet.2018.06.010.

Sema, M., A. Alemu, A. G. Bayih, S. Getie, G. Getnet, D. Guelig, R. Burton, P. Labarre, and D. R. Pillai. 2015. Evaluation of noninstrumented nucleic acid amplification by loop-mediated isothermal amplification (NINA-LAMP) for the diagnosis of malaria in Northwest Ethiopia. Malar. J. 14:44. https://doi.org/10.1186/ s12936-015-0559-9.

Singleton, J., J. L. Osborn, L. Lillis, K. Hawkins, D. Guelig, W. Price, R. Johns, K. Ebels, D. Boyle, B. Weigl, and P. LaBarre. 2014. Electricity-free amplification and detection for molecular point-ofcare diagnosis of HIV-1. PLoS One 9:e113693. https://doi.org/10 .1371/journal.pone.0113693.

Song, X., S. Shukla, G. Lee, S. Park, and M. Kim. 2016. Detection of Cronobacter genus in powdered infant formula by enzyme-linked immunosorbent assay using anti-Cronobacter antibody. Front. Microbiol. 7:1124. https://doi.org/10.3389/fmicb.2016.01124.

Sun, Y., T. L. Quyen, T. Q. Hung, W. H. Chin, A. Wolff, and D. D. Bang. 2015. A lab-on-a-chip system with integrated sample preparation and loop-mediated isothermal amplification for rapid and quantitative detection of Salmonella spp. in food samples. Lab Chip 15:1898-1904. https://doi.org/10.1039/C4LC01459F. 
Syedmoradi, L., M. Daneshpour, M. Alvandipour, F. A. Gomez, H. Hajghassem, and K. Omidfar. 2017. Point of care testing: The impact of nanotechnology. Biosens. Bioelectron. 87:373-387. https: //doi.org/10.1016/j.bios.2016.08.084.

Wang, C. H., K. Y. Lien, J. J. Wu, and G. B. Lee. 2011. A magnetic bead-based assay for the rapid detection of methicillin-resistant Staphylococcus aureus by using a microfluidic system with integrated loop-mediated isothermal amplification. Lab Chip 11:15211531. https://doi.org/10.1039/c0lc00430h.

Wang, Y., H. Li, Y. Wang, L. Zhang, J. Xu, and C. Ye. 2017. Loopmediated isothermal amplification label-based gold nanoparticles lateral flow biosensor for detection of Enterococcus faecalis and Staphylococcus aureus. Front. Microbiol. 8:192. https://doi.org/10 .3389/fmicb.2017.00192.

Wang, Y., Y. Wang, R. Lan, H. Xu, A. Ma, D. Li, H. Dai, X. Yuan, J. $\mathrm{Xu}$, and C. Ye. 2015. Multiple endonuclease restriction real-time loop-mediated isothermal amplification. J. Mol. Diagn. 17:392401. https://doi.org/10.1016/j.jmoldx.2015.03.002.

Wu, R., X. Liu, B. Guo, F. Chen, and X. Wang. 2014. Development of double loop-mediated isothermal amplification to detect Listeria monocytogenes in food. Curr. Microbiol. 69:839-845. https://doi .org/10.1007/s00284-014-0661-1.

Yan, L., J. Zhou, Y. Zheng, A. S. Gamson, B. T. Roembke, S. Nakayama, and H. O. Sintim. 2014. Isothermal amplified detection of DNA and RNA. Mol. Biosyst. 10:970. https://doi.org/10.1039/ c3mb70304e.

Yan, Q., and S. Fanning. 2015. Strategies for the identification and tracking of Cronobacter species: An opportunistic pathogen of con- cern to neonatal health. Front Pediatr. 3. https://doi.org/10.3389/ fped.2015.00038.

Yang, Y., F. Xu, H. Xu, Z. P. Aguilar, R. Niu, Y. Yuan, J. Sun, X. You, W. Lai, Y. Xiong, C. Wan, and H. Wei. 2013. Magnetic nano-beads based separation combined with propidium monoazide treatment and multiplex PCR assay for simultaneous detection of viable Salmonella Typhimurium, Escherichia coli O157:H7 and Listeria monocytogenes in food products. Food Microbiol. 34:418-424. https://doi.org/10.1016/j.fm.2013.01.004.

Yin, H. Y., T. J. Fang, and H. W. Wen. 2016. Combined multiplex loop-mediated isothermal amplification with lateral flow assay to detect sea and seb genes of enterotoxic Staphylococcus aureus. Lett. Appl. Microbiol. 63:16-24. https://doi.org/10.1111/lam.12590.

Zhao, Y., X. Jiang, Y. Qu, R. Pan, X. Pang, Y. Jiang, and C. Man. 2017. Salmonella detection in powdered dairy products using a novel molecular tool. J. Dairy Sci. 100:3480-3496. https://doi.org/ 10.3168/jds.2016-12535.

\section{ORCIDS}

Yujun Jiang (1) https://orcid.org/0000-0002-2857-2137

Sihan Chen ๑ https://orcid.org/0000-0002-5249-8723

Wei Zhang (1) https://orcid.org/0000-0003-0773-7788

Chaoxin Man ำ https://orcid.org/0000-0003-2050-4639 